\title{
A Stimulated Raman Scattering CMOS Pixel Using a High-Speed Charge Modulator and Lock-in Amplifier
}

\author{
De Xing Lioe ${ }^{1}$, Kamel Mars ${ }^{1}$, Shoji Kawahito ${ }^{1, *}$, Keita Yasutomi $^{1}$, Keiichiro Kagawa ${ }^{1}$, \\ Takahiro Yamada ${ }^{2}$ and Mamoru Hashimoto ${ }^{2}$ \\ 1 Research Institute of Electronics, Shizuoka University, 3-5-1 Johoku, Nakaku, Hamamatsu, \\ Shizuoka 432-8011,Japan; lioe@idl.rie.shizuoka.ac.jp (D.X.L.); kamel@idl.rie.shizuoka.ac.jp (K.M.); \\ kyasu@idl.rie.shizuoka.ac.jp (K.Y.); kagawa@idl.rie.shizuoka.ac.jp (K.K.) \\ 2 Graduate School of Engineering Science, Osaka University, 1-3 Machikaneyama, Toyonaka, \\ Osaka 560-8531, Japan; yamada@sml.me.es.osaka-u.ac.jp (T.Y.); mamoru@me.es.osaka-u.ac.jp (M.H.) \\ * Correspondence: kawahito@idl.rie.shizuoka.ac.jp; Tel.: +81-53-478-1313
}

Academic Editor: Vittorio M. N. Passaro

Received: 4 March 2016; Accepted: 6 April 2016; Published: 13 April 2016

\begin{abstract}
A complementary metal-oxide semiconductor (CMOS) lock-in pixel to observe stimulated Raman scattering (SRS) using a high speed lateral electric field modulator (LEFM) for photo-generated charges and in-pixel readout circuits is presented. An effective SRS signal generated after the SRS process is very small and needs to be extracted from an extremely large offset due to a probing laser signal. In order to suppress the offset components while amplifying high-frequency modulated small SRS signal components, the lock-in pixel uses a high-speed LEFM for demodulating the SRS signal, resistor-capacitor low-pass filter (RC-LPF) and switched-capacitor (SC) integrator with a fully CMOS differential amplifier. AC (modulated) components remained in the RC-LPF outputs are eliminated by the phase-adjusted sampling with the SC integrator and the demodulated DC (unmodulated) components due to the SRS signal are integrated over many samples in the SC integrator. In order to suppress further the residual offset and the low frequency noise ( $1 / \mathrm{f}$ noise) components, a double modulation technique is introduced in the SRS signal measurements, where the phase of high-frequency modulated laser beam before irradiation of a specimen is modulated at an intermediate frequency and the demodulation is done at the lock-in pixel output. A prototype chip for characterizing the SRS lock-in pixel is implemented and a successful operation is demonstrated. The reduction effects of residual offset and $1 / \mathrm{f}$ noise components are confirmed by the measurements. A ratio of the detected small SRS to offset a signal of less than $10^{-5}$ is experimentally demonstrated, and the SRS spectrum of a Benzonitrile sample is successfully observed.
\end{abstract}

Keywords: stimulated Raman scattering; CMOS image sensor; lock-in amplifier; low frequency noise; double modulation; Raman shift

\section{Introduction}

Developments in optical imaging techniques enable better understanding of the microscopic world. One of the modalities, Raman scattering, has long been explored to provide a contrast mechanism for label-free, noninvasive imaging to study biological samples by detecting specific vibrational spectra of chemical bonds [1,2]. Spontaneous Raman scattering microscopy possesses the relatively high spatial resolution capability. However, due to the extremely small Raman cross section, the spontaneous Raman scattering microscopy requires a lengthy acquisition time and thus is not suitable for live imaging [3]. Coherent Raman scattering (CRS) microscopy enhances the Raman response significantly, which facilitates high-speed imaging. The CRS utilizes two laser sources, pump beam at frequency $\omega_{\mathrm{p}}$ and Stokes beam at frequency $\omega_{\mathrm{s}}$, to coincide on the sample. When the 
frequency difference matches a specific molecular vibrational frequency, interaction between two laser beams and molecular vibration occurs in the focal volume. There are two CRS techniques: coherent anti-Stokes Raman scattering (CARS) and stimulated Raman scattering (SRS). The CARS produces anti-Stokes emission at $\omega_{a s}=2 \omega_{p}-\omega_{s}$ by virtue of a wave-mixing process. However, CARS suffers from non-resonant background signal, which interferes with the resonant signal and consequently causes the CARS spectrum to be distorted [4,5]. A number of approaches have been carried out to extract only the quantitative data out of CARS [6]. Nonetheless, these complicate the experimental system and analysis.

In contrast to the CARS, the SRS signal is stimulated only when the frequency difference, $\Omega=\omega_{p}-\omega_{s}$, matches the molecular vibrational frequency. SRS does not exhibit non-resonant background and can be interpreted directly. The SRS signal occurs at the same frequency, and slightly varies in intensity, as the incident laser source. In order to detect this weak SRS signal, which is on the order of $10^{-4}$ to $10^{-5}$ of the offset due to the pump laser source, and a lock-in detection is typically used with a single photodiode [7-9]. However, such a method restricts the SRS from performing multi-point parallel measurement, which relaxes damages to a biological specimen. An alternative method of utilizing a fast CMOS array has been demonstrated [10] but with slow modulation rate.

Here, we present an approach with CMOS-based SRS lock-in pixels in order to address the limitation of currently available technology. Instead of adopting a separate photodiode and lock-in amplifier, we implemented both components in each pixel to extract the weak SRS signal from a huge background. The proposed pixel structure uses a photo-charge modulator based on a lateral electric field control to achieve high-speed demodulation of SRS signal components. Through a low-pass filter section, the small demodulated components are sampled with a switched-capacitor (SC) integrator and are integrated over many samples in an SC integrator to amplify the signal while eliminating the residual offset components. An SRS CMOS image sensor chip for the proof of concept is implemented and a successful operation is demonstrated.

The remainder of this paper describes the proposed image sensor and measurement results in detail. Section 2 describes the structure and operations of the SRS pixel. Section 3 explains the 1/f noise reduction technique utilized in the lock-in amplifier. The implementation and measurement results are shown in Section 4, followed by conclusions in Section 5.

\section{Demodulator and Readout Circuits in SRS Lock-in Pixels}

\subsection{SRS Signal and Lock-in Pixel}

The principle of SRS is illustrated as an energy diagram in Figure 1. If the frequency difference between pump and Stokes pulses matches a particular molecular vibrational frequency, and energy transfer happens between the two laser pulses, where picosecond laser pulses with high peak power are usually used for SRS because SRS is nonlinear optical phenomena. The Stokes beam is modulated, and, consequently, the intensity of the resulting pump is varied, as shown in Figure 2. The SRS signal, which is determined by the ratio of the intensity variation $\left(\Delta I_{p}\right)$ to the intensity of pump $\left(I_{p}\right), \Delta I_{p} / I_{p}$ is very weak $\left(<10^{-4}\right)$. Detection of such a small signal is a big challenge because photon shot noise of the pump laser is typically greater than the interested SRS signal if the observation time is not enough and the intensity of the lasers fluctuates. Despite that, high frequency $(>1 \mathrm{MHz})$ of modulation and detection with sufficient observation time can effectively reduce the effect of the laser noise, such as $1 / \mathrm{f}$ noise and laser fluctuation, which generally appears at low frequencies $[5,11]$. 


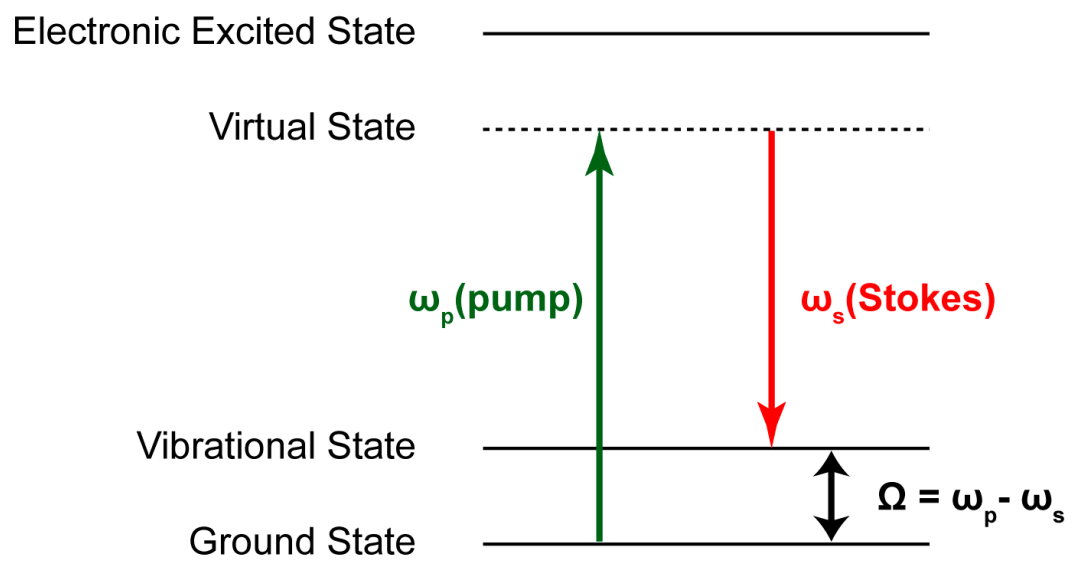

Figure 1. Energy bands in stimulated Raman scattering (SRS).

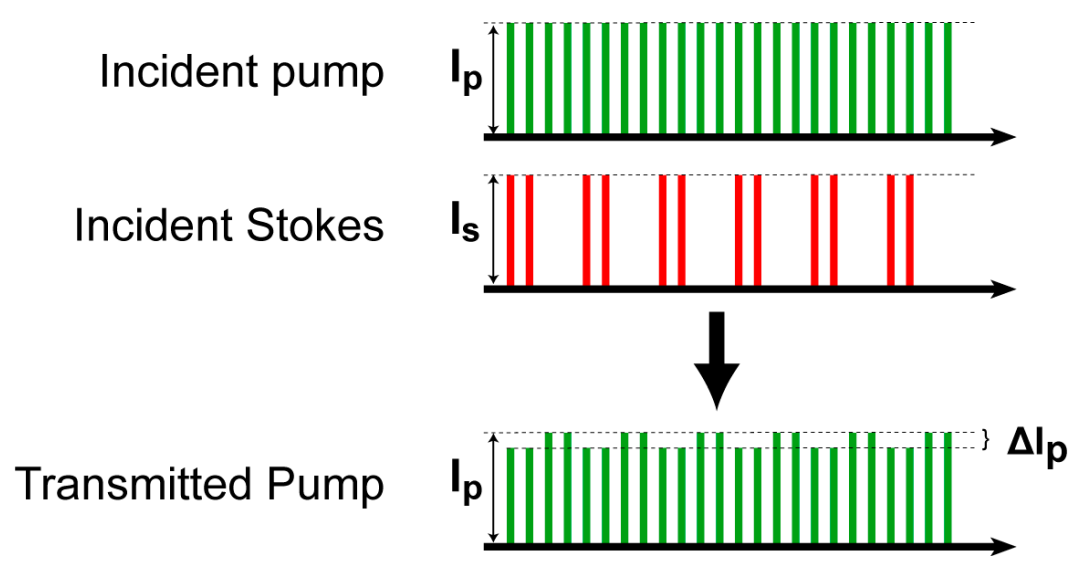

Figure 2. High-speed modulation for small SRS signal.

A general configuration for a lock-in amplifier for detecting a small AC modulated signal in a large offset is shown in Figure 3. The AC modulated signal at around frequency $f_{m}$ is demodulated and converted to a signal at around DC and the DC offset of the input is modulated and converted to an $\mathrm{AC}$ component at $\mathrm{f}_{\mathrm{m}}$ by a demodulator. A small DC signal component only appears at a low-pass filter (LPF) output if the LPF can suppress the AC component of the demodulator output. The small DC signal component is integrated over multiple samples, which is essentially an averaging, to reduce the photon shot noise and the other noises that may be superimposed in it due to the succeeding circuits and systems.

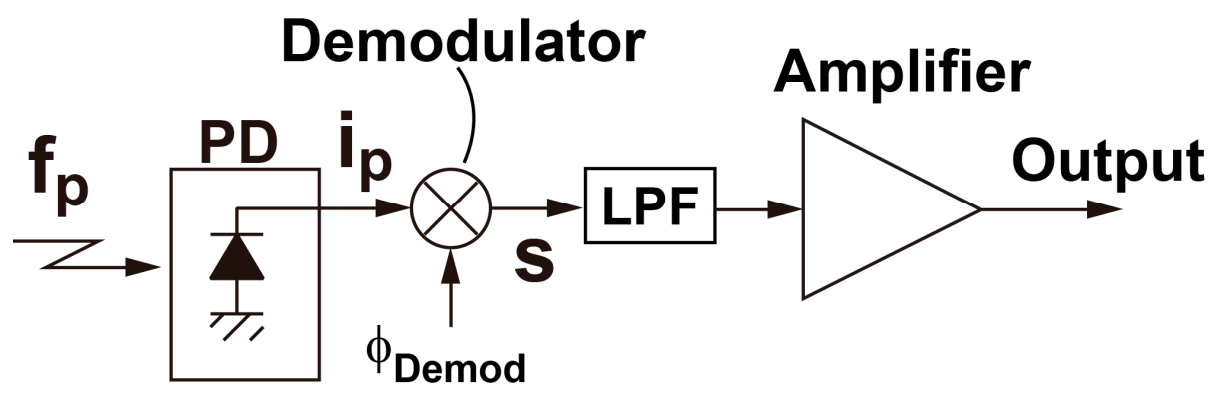

(a)

Figure 3. Cont. 


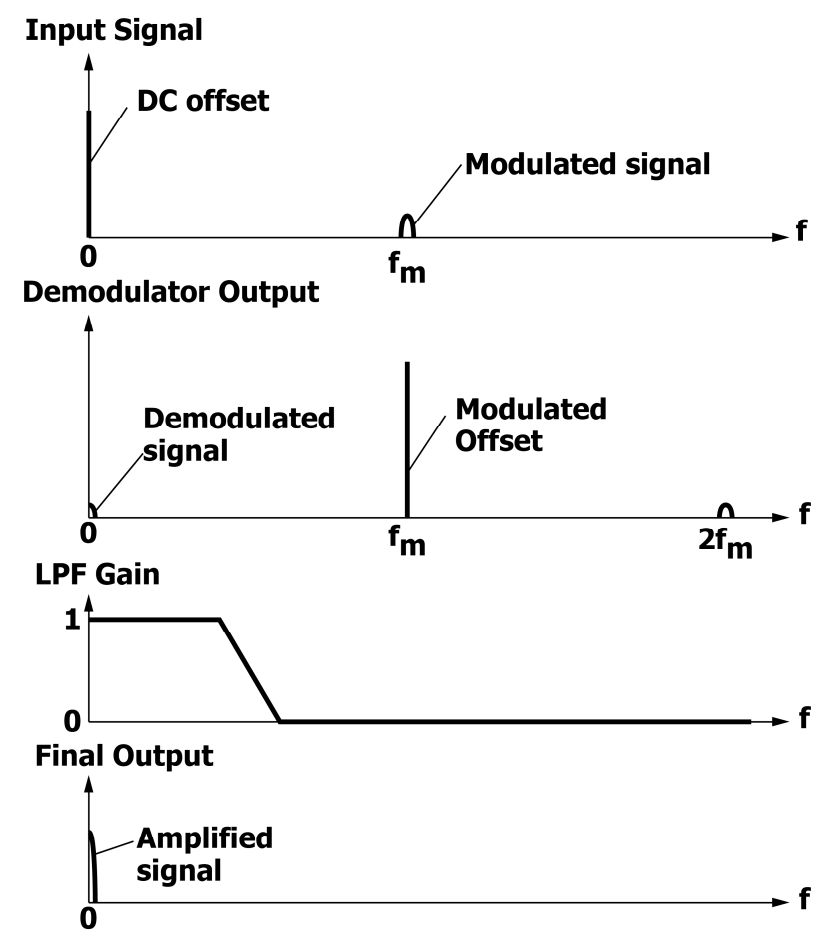

(b)

Figure 3. Lock-in amplifier for detecting small signal with large offset: (a) block diagram; (b) operation in frequency domain.

\subsection{Lock-in Pixel Design for AC Signal Detection in Large DC Offset}

A conceptual circuit diagram for implementing the lock-in pixel for a small AC signal detection in a large DC offset (unmodulated pulse train) is shown in Figure 4a. The operation of the lock-in pixel if the input signal is a pulse-modulated AC signal with continuous DC offset is shown in Figure 4b. The function of the demodulator, which converts the AC modulated signal into a signal at around DC, is expressed as:

$$
\left\{\begin{array}{l}
I_{i p}=I_{p}, I_{i n}=0\left(\varnothing_{\text {Demod }}=" 1^{\prime \prime}\right) \\
I_{i p}=0, I_{\text {in }}=I_{p}\left(\varnothing_{\text {Demod }}=" 0^{\prime \prime}\right)
\end{array}\right.
$$

where $I_{p}$ is the photo current of the photo detector, $I_{i p}$ and $I_{i n}$ are output currents of the two-tap demodulator, and $\varphi_{\text {Demod }}$ is the demodulation clock. If the cutoff frequency of the LPF is much smaller than the modulation clock frequency, the LPF outputs contain DC components consisting of the DC offset and the demodulated signal. The difference of the two outputs contains the demodulated signal only and is amplified by the amplifier section.

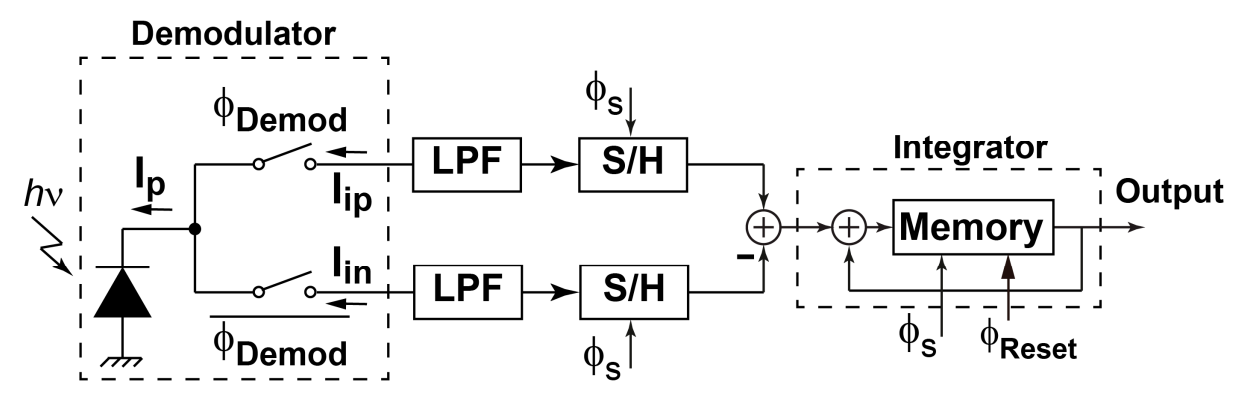

(a)

Figure 4. Cont. 


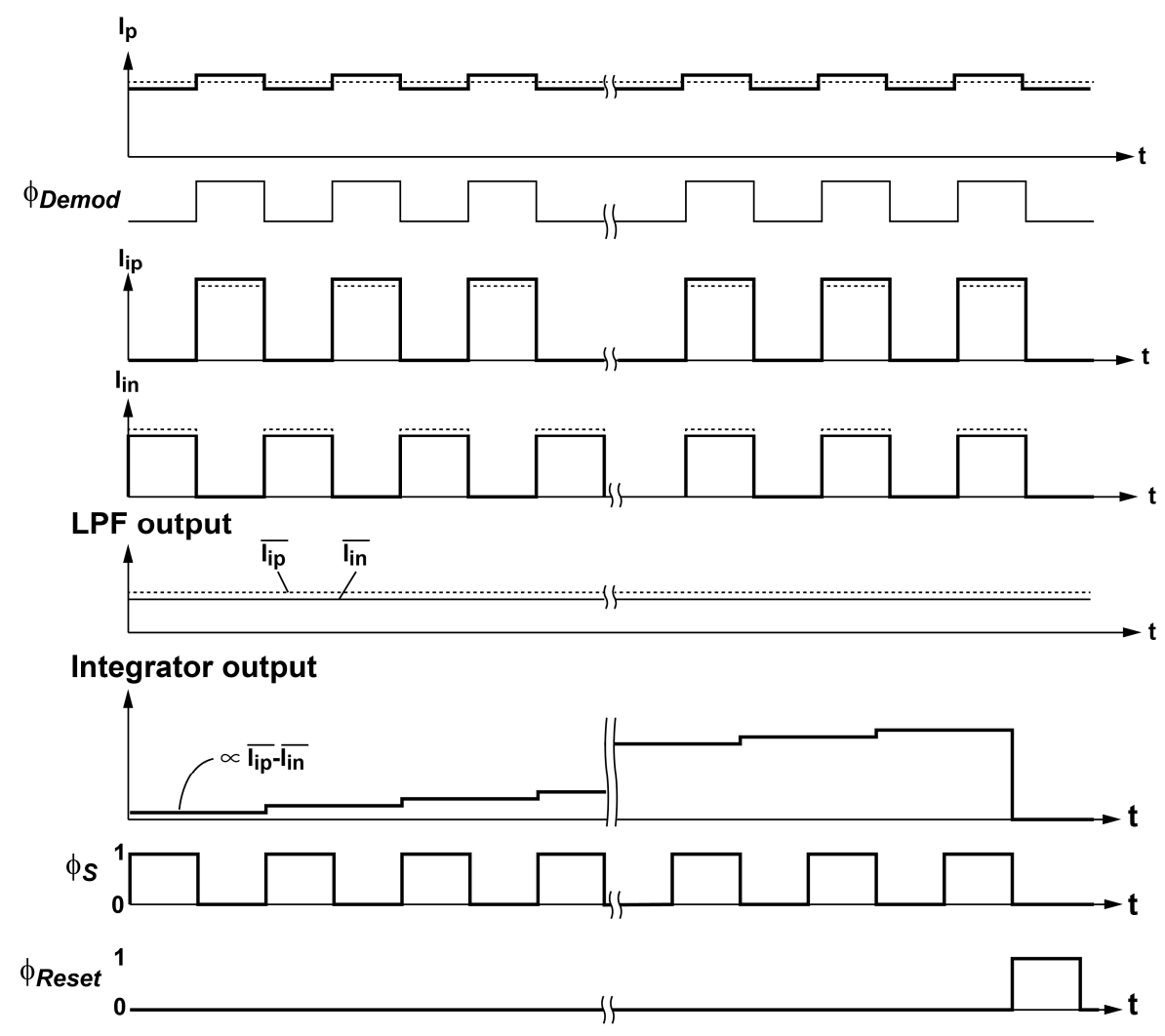

(b)

Figure 4. Lock-in Pixel Using a Two-Tap Demodulator: (a) block diagram (b) operation of the lock-in pixel.

\subsection{Demodulator Design}

The high-speed demodulator, which is expressed as a set of two switches in Figure 4a can be implemented with gated photo-detectors [11]. To implement the high-speed demodulator using a standard CMOS image sensor technology with a pinned photodiode option, the authors have proposed a new type of photo-charge modulators, a so-called lateral electric field modulator (LEFM) [12]. Figure 5 shows the top view of a two-tap LEFM used in the lock-in pixel for SRS signal detection. The two-tap LEFM has a pinned photodiode with a set of three gates $G_{1}, G_{2}$ and $G_{3}$ to apply and control the lateral electric field of $\mathrm{X}-\mathrm{X}^{\prime}$ direction in the pinned photodiode by the modulation of its depleted potential. In the central gate $\mathrm{G}_{3}$, a middle constant voltage (e.g., $0 \mathrm{~V}$ ) is applied. Small positive voltage (e.g., $2 \mathrm{~V}$ ) or negative voltage (e.g., $-1.3 \mathrm{~V}$ ) are used for $\mathrm{G}_{1}$ and $\mathrm{G}_{2}$ to control the lateral electric field of $\mathrm{X}-\mathrm{X}^{\prime}$ direction. As shown in Figure $5 b$, the application of high $(\mathrm{H})$ and low $(\mathrm{L})$ voltage levels to $\mathrm{G}_{1}$ and $\mathrm{G}_{2}$, respectively, creates potential profiles for transferring photo electrons to the left and application of $L$ and $H$ to $G_{1}$ and $G_{2}$, respectively, creates the potential profiles for transferring to the right. The mechanism of the modulation of depleted potential in the pinned photodiode can be explained by Figure $5 c$ which shows the cross-section and potential profile of $Y-Y^{\prime}$ direction. For instance, by applying a low voltage (e.g., $-1.3 \mathrm{~V}$ ) in the gate $\mathrm{G}_{1}$, the potential under the gates is lowered to around $0 \mathrm{~V}$ and a resulting voltage of the pinned diode is set to relatively low voltage. When a relatively high voltage (e.g., $2 \mathrm{~V}$ ) is applied to the gates, the potential under the gate and the resulting potential in the pinned diode are raised to high because of the modulation of fringing electric field due to the applied gate voltage change of the set of gates $G_{1}$. The LEFM ensures a very high-speed electron transfer because this structure does not have a problem of creation of potential barrier at the edge of the transfer gate and charge trapping under the gate $\left(\mathrm{Si}-\mathrm{SiO}_{2}\right.$ interface) unlike conventional transfer technique using a transfer gate in the signal path. 

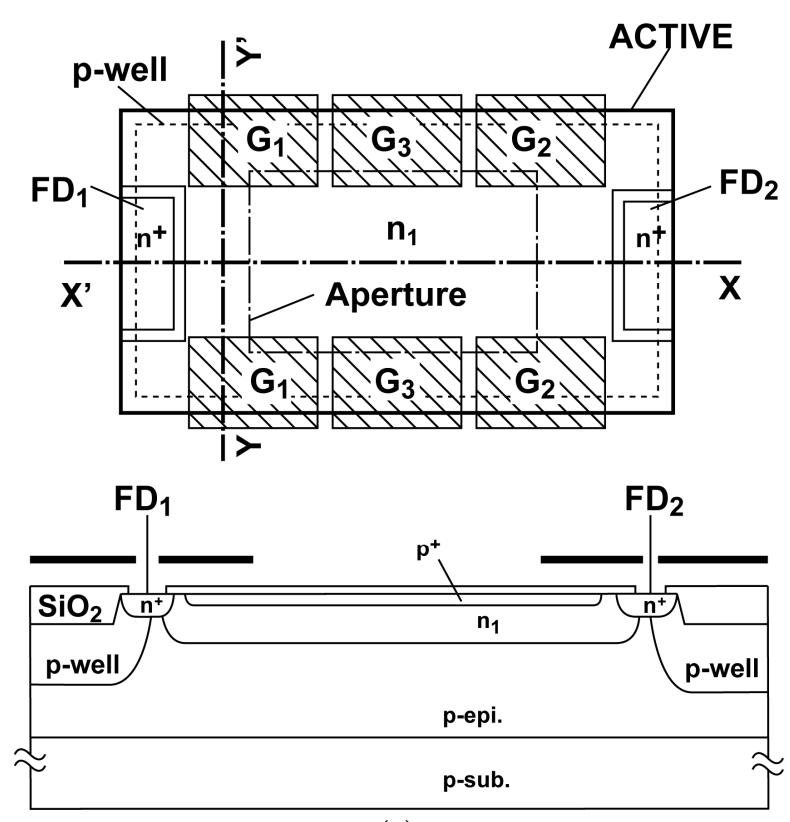

(a)

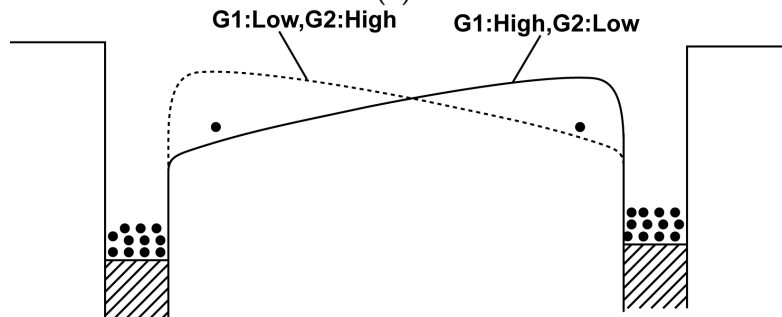

(b)

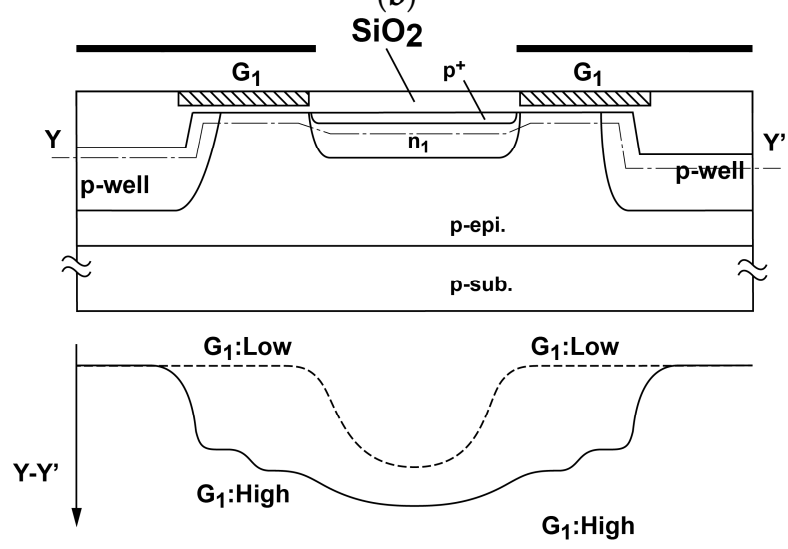

(c)

Figure 5. Lateral Electric Field Charge Modulator (LEFM): (a) top view and cross-sectional view for $X-X^{\prime}(\mathbf{b})$ potential profiles for photo-electrons transfer (c) cross-sectional view and potential profile for $Y-Y^{\prime}$.

In order to investigate the characteristics of the used LEFM detector shown in Figure 5, a simulation has been conducted using a device simulator SPECTRA. The implementation using $0.11 \mu \mathrm{m}$ CMOS image sensor (CIS) technology with a lightly-doped substrate is assumed, and device parameters based on this technology are used for the device simulation. A simulation result of the potential profile along the transfer channel in the LEFM is shown in Figure 6. In this simulation, the potential profiles for the cases $G_{1}$ and $G_{2}$ are set to $-1.3 \mathrm{~V}$ and $2 \mathrm{~V}$, respectively, and $\mathrm{G}_{1}$ and $\mathrm{G}_{2}$ are set to $2 \mathrm{~V}$ and $-1.3 \mathrm{~V}$, respectively, are shown. The gate voltage of $\mathrm{G}_{3}$ is set to always $0 \mathrm{~V}$. By using a $3.3 \mathrm{~V}(-1.3 \mathrm{~V}$ to $2 \mathrm{~V})$ for gate driving voltage difference between $G_{1}$ and $G_{2}$, a maximum potential 
modulation of approximately $124 \mathrm{mV}$ is created for the distance of $2.6 \mu \mathrm{m}$ resulting in a high electric field along the $\mathrm{X}$ coordinate. This electric field allows us to have a photo-electron transfer time of less than one nanosecond and is sufficient for the demodulation clock of $20 \mathrm{MHz}$ in this design.

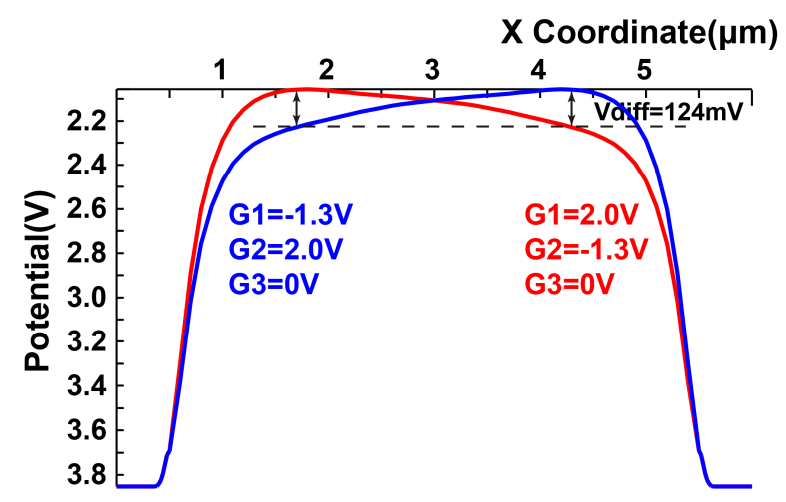

Figure 6. Simulation result of potential profile along $X$ coordinate.

In the actual pixel design, a $10 \times 10$ array of the LEFM units where all the corresponding outputs are connected in parallel is used in each pixel as shown in Figure $7 \mathrm{~b}$.

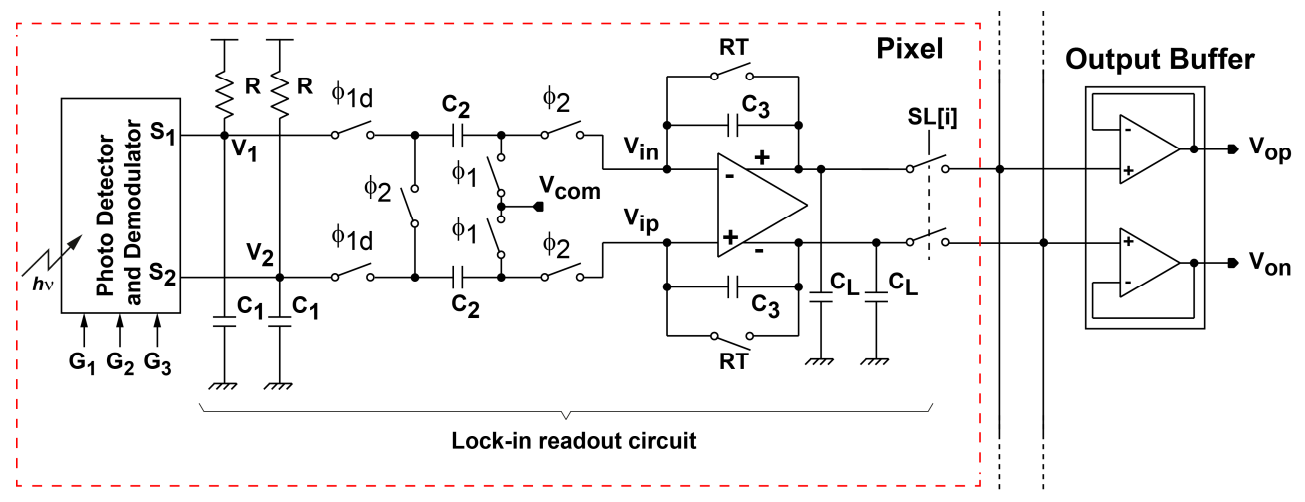

(a)

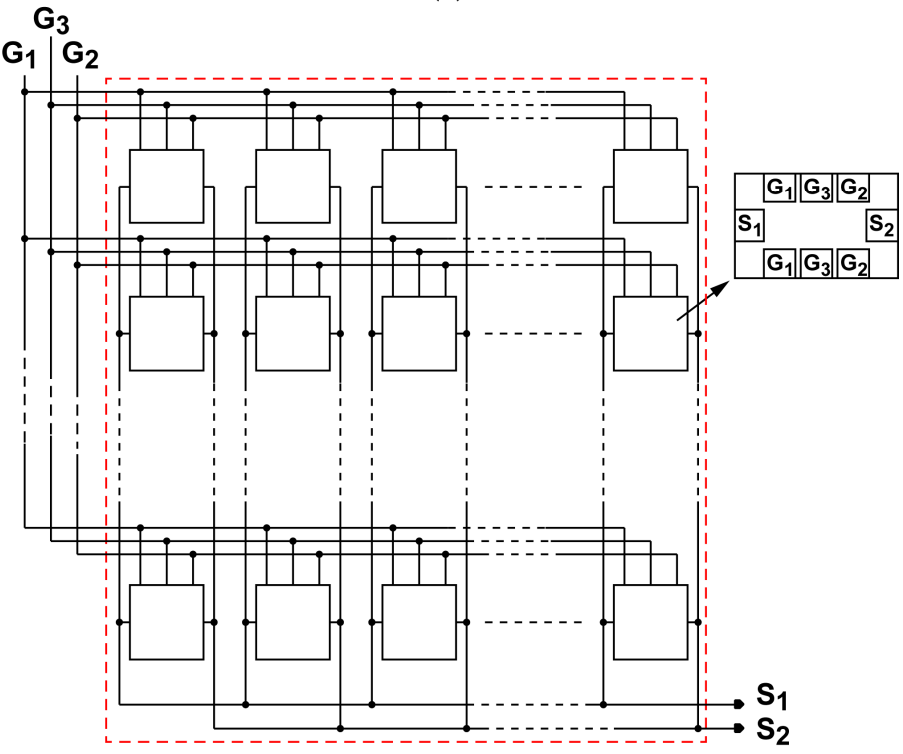

(b)

Figure 7. Lock-in pixel readout circuit: (a) lock-in readout circuit (b) photo detector and demodulator. 


\subsection{Pixel Readout Circuit Design and Operation}

The readout circuit together with the photo detector and demodulator for the SRS lock-in pixel imager is depicted as shown in Figure 7. The LPF for extracting the demodulated SRS signal is implemented with a 1st-order RC filter. The sampling of the LPF outputs and discrete-time integration of the difference of the sampled LPF output signals are implemented with a fully-differential switched-capacitor (SC) integrator. The timing chart for the operation of the lock-in pixel readout circuits and waveform of the LPF outputs are shown in Figure 8. The timing $G_{1}$ and $G_{2}$, which controls the demodulation of the two-tap LEFM, are appropriately set to sample the DC signal exactly during the Stokes on and Stokes off, respectively The phase diagram of the readout circuits is shown in Figure 9. Initially the integrator is reset by turning on the switches controlled by RT, $\varphi_{1}$, and $\varphi_{2}$ as shown in Figure 9a. After the integrator is reset, the LPF outputs are amplified by the SC integrator by two steps. First the LPF outputs are sampled by the equivalent circuit shown in Figure $9 \mathrm{~b}$ when switches controlled by $\varphi_{1}$ and $\varphi_{1 \mathrm{~d}}$ are turned on, and then the sampled differential charges stored in two capacitors of $C_{2}$ in the sampling phase are transferred to $C_{3}$ by using the equivalent circuit shown in Figure $9 \mathrm{c}$ when the switches controlled by $\varphi_{2}$ are turned on. The operation with the sampling (Figure 9b) and charge transfer (Figure 9c) gives one cycle of the discrete-time integration, and the gain of the SC integrator is given by the number of cycles. A fully-differential folded-cascode operational amplifier is used in the readout circuit, as shown in Figure 10. $V_{\text {in }}$ and $V_{\text {ip }}$ are the differential inputs, $V_{\text {on }}$ and $V_{o p}$ are the differential outputs, while $V_{b n}$ and $V_{b p}$ are the biasing voltages for the operational amplifier. The phase margin of the operational amplifier is 79 degrees.

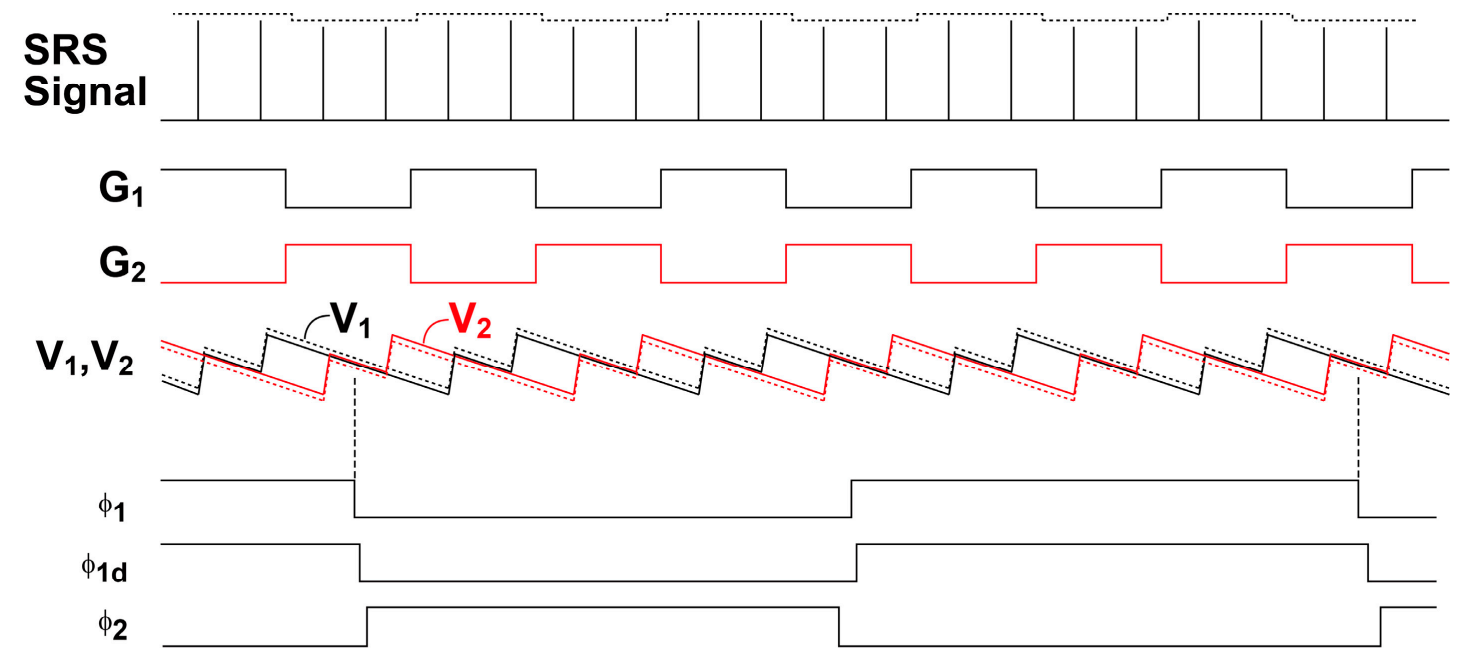

Figure 8. Timing diagram for the lock-in pixel operations.

To pick up and amplify the small demodulated signal while suppressing the large modulated DC offset, the sampling timing is very important. To find the best timing to minimize the offset, the detailed waveform after the demodulation and low-pass filtering must be considered. The actual offset signal, which is called DC offset here, is a pulse train whose amplitude is unmodulated. The waveform after the low-pass filter becomes a solid-line of $\mathrm{V}_{1}$ and $\mathrm{V}_{2}$ is shown in Figure 8. If the input light pulse train is modulated by an AC signal, the waveform becomes the dashed-line of $\mathrm{V}_{1}$ and $\mathrm{V}_{2}$ in Figure 8 . By choosing the timing of sampling pulses, $\varphi_{1}$ and $\varphi_{1 \mathrm{~d}}$ as shown in Figure 8 , it is understood that the residual DC offset is minimized and the demodulated AC signal is detected and amplified by the integrator. With the capability of extracting the SRS signal in the pixel, parallel detection of the SRS signal in a line sensor for an SRS spectrum or an area sensor for an SRS image is feasible. 


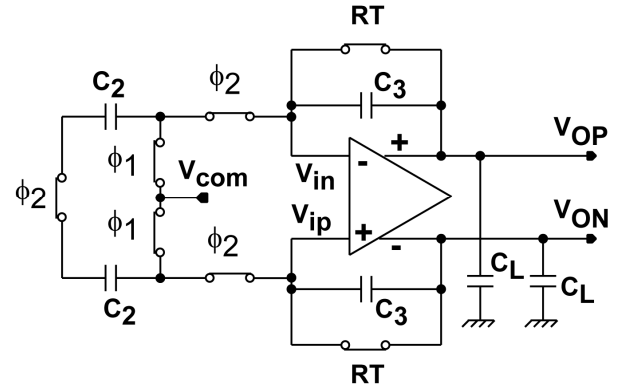

(a)

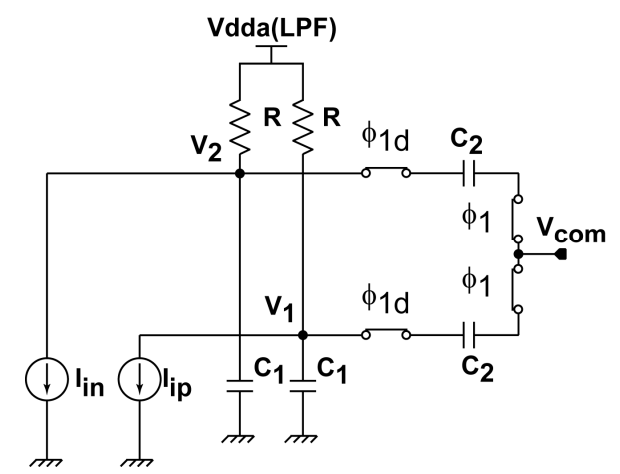

(b)

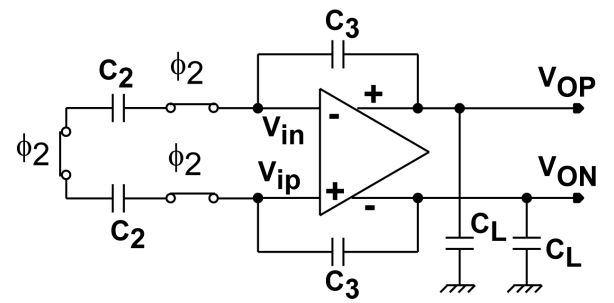

(c)

Figure 9. Phase diagrams of the lock-in readout circuits: (a) reset phase; (b) sampling phase (c) transfer phase.

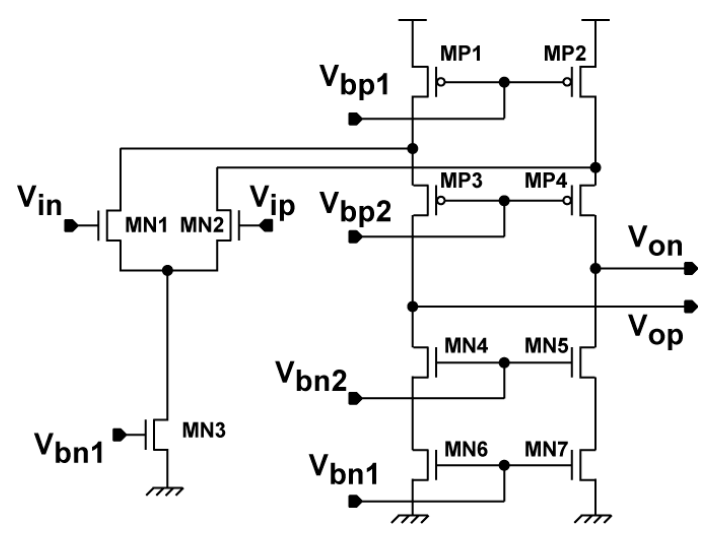

Figure 10. Schematic diagram of the fully differential folded-cascode operational amplifier.

\section{1/f Noise Reduction of a Lock-in Amplifier}

Residual offset and low-frequency noise (1/f noise) are reduced in two steps using the double modulation technique where the principle is depicted in Figure 11. For further detailed description of the double modulation technique, the fact that the laser light is actually a high-frequency pulse train whose frequency is four times the modulation frequency should be considered in the frequency spectrum, but such high-frequency components are omitted in Figure 11 for simplicity. The LPF in the lock-in pixel is not sufficient to fully attenuate the DC offset. Therefore, the phase delay between the demodulated pixel output signal and the readout sampling signals $\varphi_{1}, \varphi_{1 d}$ and $\varphi_{2}$ should be carefully adjusted to ensure that the offset signal is canceled out or reduced to the minimum in order to achieve a high dynamic range. If there is no light or just a DC light, in fact, light pulses are used but the amplitude of pulses is unmodulated, is applied to the sensor, and the amplifier differential output should be reduced to be as small as possible by a careful choice of the phase delay value. The double modulation technique is then used to reduce the low-frequency noise component and the residual offset component, which are also introduced by the lock-in pixel circuitry. Initially, AC light signal 
(SRS signal) with the modulation frequency of $f_{s}$ is multiplied by the square wave signal of frequency $f_{c}$ through a function generator in order to change its polarity at every half cycle of the square wave. After this first modulation, the AC light signal has mainly two frequency components $f_{s}-f_{c}$ and $f_{s}+f_{c}$ and their harmonics. The DC light signal (unmodulated pump laser pulses) and AC light (modulated Stokes laser pulses) irradiate the specimen and the output from the specimen, the modulated pump laser, is received by the lock-in pixels. The SRS signal due to molecular vibration is observed from the specimen, and it results in the modulation at the frequency components $f_{S}-f_{\mathcal{C}}$ and $f_{S}+f_{c}$. The lock-in pixel, which is locked at the frequency $f_{\mathrm{s}}$, demodulates and amplifies the SRS signal at the pixel output. The SRS signal modulated at frequencies $\mathrm{f}_{\mathrm{c}}$ and its odd harmonics are produced. The pixel output is converted to a digital number using an A-to-D converter in the external system. The demodulation of the modulated SRS signal at the frequency $f_{c}$ is done in the digital domain by multiplying a square wave with the frequency of $f_{c}$ and that takes 1 or -1 , and then applying a digital low-pass filter. This demodulation process shifts the low-frequency noise components due to the $1 / \mathrm{f}$ noise of the lock-in amplifier and residual offset to a higher frequency around $f_{c}$ and its harmonics, and the shifted noise and residual offset are suppressed by applying the digital low-pass filter. Although white noise increases when $1 / \mathrm{f}$ noise is reduced, white noise can then be reduced by averaging and increasing the time of observation. The same cannot be done for $1 / \mathrm{f}$ noise.

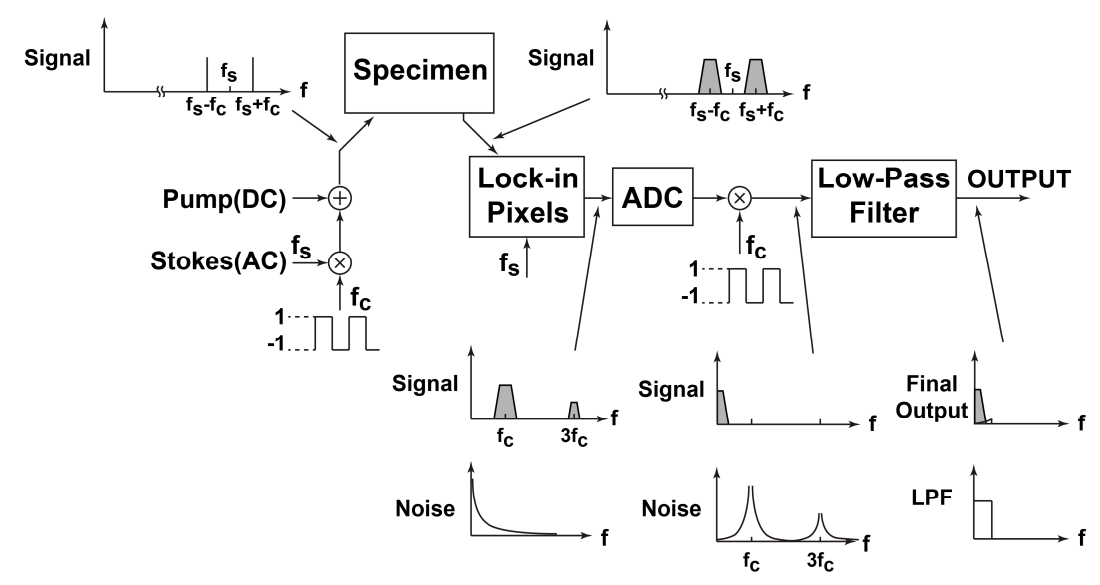

Figure 11. Double modulation technique to reduce low-frequency noise component and residual offset component.

\section{Implementation and Measurement Results}

\subsection{Implementation of CMOS SRS Pixel Array Chip}

An SRS CMOS imager chip has been fabricated using $0.11 \mu \mathrm{m}$ CMOS image sensor process technology to experimentally evaluate the performances of the proposed circuit. Figure 12 shows the layout of the SRS CMOS pixel. A photo receiver with $10 \times 10$ sub-array of demodulators and lock-in readout circuits are put into the pixel area of $100.8 \mu \mathrm{m} \times 100.8 \mu \mathrm{m}$. The entire chip consists of an array of $64 \times 8$ pixels, a pixel selector and buffer amplifiers. As a preliminary study for the operation as an SRS imager, only a single pixel is characterized.

\subsection{Experimental Setup and Measurement Conditions}

Figure 13a shows the simplified illustration of the experimental setup to characterize the lock-in pixel of the proposed SRS imager. Two laser sources are used; one is the master laser, also called a pump laser to generate the unmodulated (DC) laser light running at $80 \mathrm{MHz}$ with a fixed wavelength of $709.5 \mathrm{~nm}$, and the other is the tunable slave laser, also called a Stokes laser to generate the modulated (AC) laser light running at $20 \mathrm{MHz}$ where the wavelength is initially fixed at $837 \mathrm{~nm}$. The Stokes 
laser wavelength can be tuned using the computer controlled acousto-optic tunable filters (AOTF) for SRS spectrum measurement requirements. The proposed imager is incorporated to the lock-in camera controlled by a computer and being synchronized to the function generator. The other channel of the function generator is triggered by the lock-in camera to change the polarity at each frame in order to achieve the double modulation technique. The experimental setup includes also a balanced cross-correlator for synchronization of pump and Stokes lasers and an electro-optic modulator (EOM) for modulating Stokes laser beam [13]. Figure 13b shows the lens setup for further works of an array of pixels to be used to obtain the image, with other experimental setups being identical. The microlens array [14] splits laser beams into multiple collimated beamlets to be focused on multiple spots of the specimen. The multi-points SRS signal will then be detected in parallel with the proposed SRS imager. Besides the use of an array of pixels and parallel detection, $x-y$ stage scanning will also be utilized to obtain a high-definition image. If a large density detector is accomplished, high-speed imaging could be realized by rotating the microlens array disk for scanning the foci [14]. The printed circuit board (PCB) sensor board and chip micrograph are shown in Figure 14. Figure 15 shows the photograph of the real measurement setup for SRS with the developed CMOS imager sensor board mounted on top of the microscope.

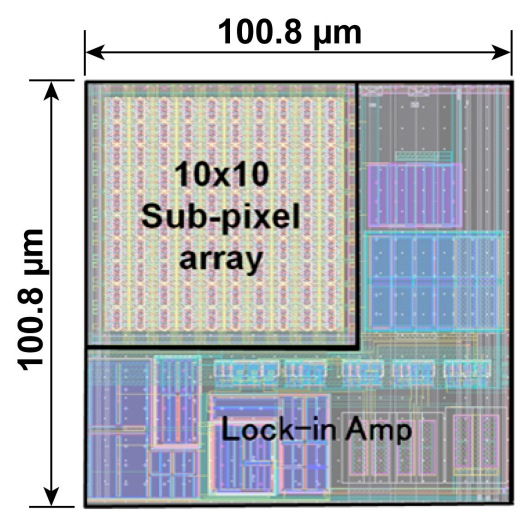

Figure 12. SRS pixel layout showing the sub-pixel array and lock-in amplifier.

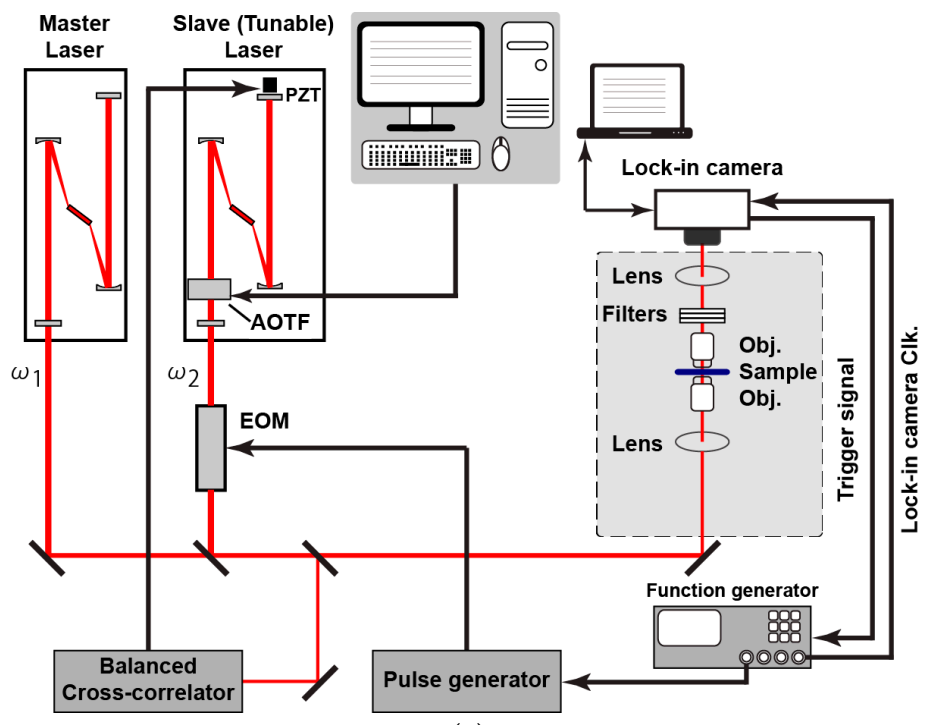

(a)

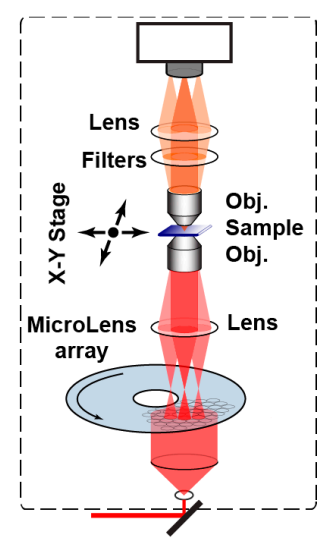

(b)

Figure 13. Experimental setup for SRS: (a) the setup used to characterize the pixel; (b) the lens setup to be used for image sensor array. 


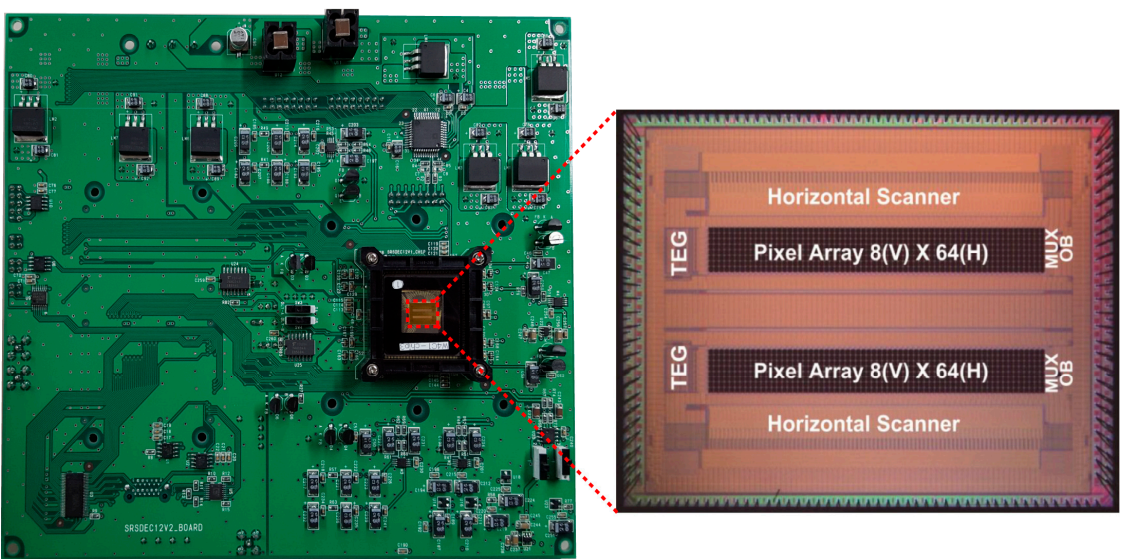

Figure 14. Photograph of the PCB sensor board with micrograph of the prototype SRS CMOS imager. The chip consists of $64 \times 8$ pixel array, horizontal scanner as pixel selector, and output buffer amplifiers.

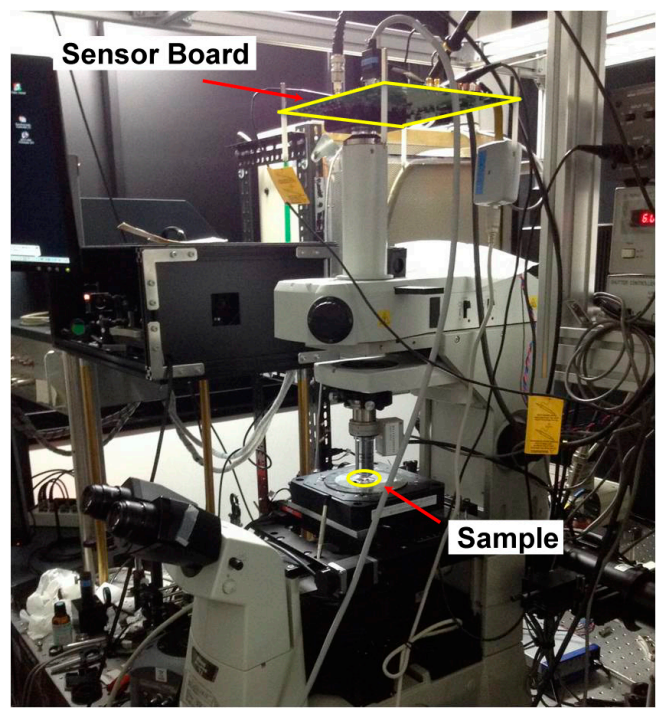

Figure 15. Photograph of the measurement setup for SRS.

The measurement conditions for the following results are shown in Table 1. The pump and Stokes laser powers are $3.8 \mathrm{~mW}$ and $8.0 \mathrm{~mW}$, respectively. Pump laser wavelength is fixed at $709.5 \mathrm{~nm}$ while Stokes laser wavelength is swept from $837 \mathrm{~nm}$ to $846 \mathrm{~nm}$ with step size of $0.25 \mathrm{~nm}$. The sampling at the frequency of $5 \mathrm{MHz}$ during the integration time of $150 \mu$ s yields a gain of 750 . The sensitivity of the pixel for quantum efficiency of $40 \%$ at $800 \mathrm{~nm}$ is $0.258 \mathrm{~A} / \mathrm{W}$. realized

Table 1. Measurement conditions.

\begin{tabular}{cc}
\hline Parameter & Value \\
\hline AC Power & $3.8 \mathrm{~mW}$ \\
DC Power & $8.0 \mathrm{~mW}$ \\
Pump laser wavelength & $709.5 \mathrm{~nm}$ \\
Stokes laser wavelength & $837 \sim 846 \mathrm{~nm}$ \\
Step size & $0.25 \mathrm{~nm}$ \\
Modulation frequency & $20 \mathrm{MHz}$ \\
Integrator sampling frequency & $5 \mathrm{MHz}$ \\
Integration time & $150 \mu \mathrm{s}$ \\
Number of integration (Gain) & 750 \\
\hline
\end{tabular}




\subsection{Measurement Results}

For SRS measurement, the pump beam is separated from the modulated Stokes beam using optical filters, and the modulation appearing on pump beam is detected. Here, for evaluation of the lock-in pixel performance, DC pump laser pulses and the AC Stokes laser pulses are input into the pixel to stimulate the SRS signal.

As described in Section 2.4, the phase delay between the demodulator clock and the readout sampling clocks $\varphi_{1}, \varphi_{1 d}$ and $\varphi_{2}$ must be carefully tuned to minimize the residual offset in the pixel output. The optimal phase can be found where the amplifier differential output when a DC laser is applied has approximately the same value when no light input is applied. Figure 16 shows the measurement results of the pixel outputs for a DC light and no light input as a function of the common phase delay of sampling clocks $\varphi_{1}, \varphi_{1 d}$ and $\varphi_{2}$. Because of the coupling of gating clock noise for the demodulator to analog circuits of the lock-in pixel, the output with no light also has a large variation to time. The best phase delay to meet the abovementioned condition is around $100 \mathrm{~ns}$. The following measurement results use the delay of $100 \mathrm{~ns}$.

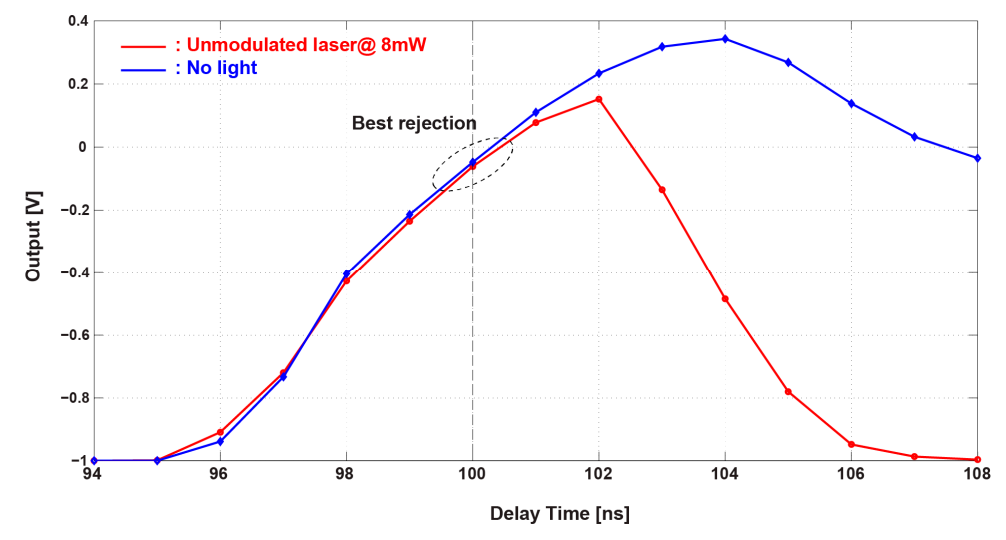

Figure 16. Delay time adjustment to minimize the residual offset in the pixel output.

To maximize the demodulation sensitivity to the SRS signal, the phase of the demodulator clock to the AC laser light must be adjusted. Figure 17 shows the lock-in pixel output as a function of Stokes laser phase change. The differential output after A/D conversion with the unit of least significant bit (LSB) is shown. Maximum output values are obtained at $150^{\circ}$ and $330^{\circ}$. This shows that there is a phase difference of $180^{\circ}$ between the two maximum values of the differential output signal. In the following measurements, the phase of the AC-modulated laser is set at $330^{\circ}$ to maximize the sensitivity.

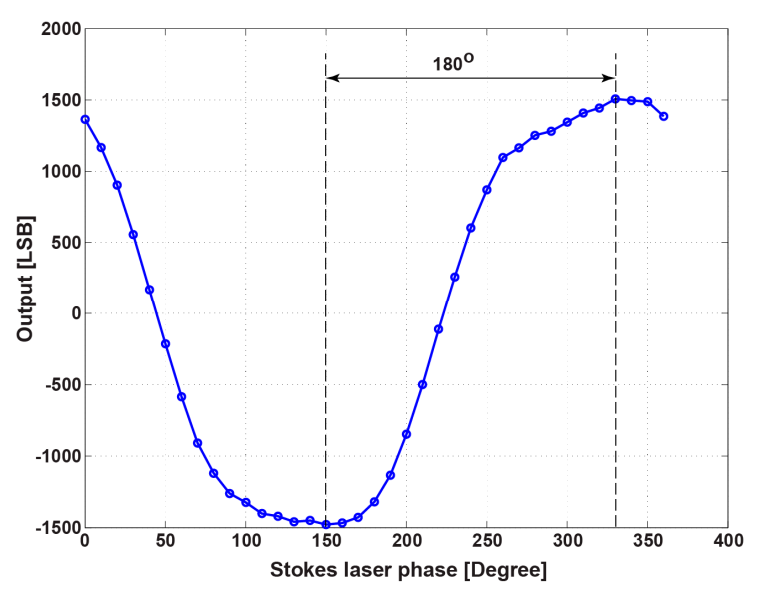

Figure 17. Pixel output as a function of the laser modulation. 
In order to demonstrate the effectiveness of the double modulation technique to reduce the residual offset signal and low frequency noise, two different noise measurements were done, with and without the double modulation technique. Figure 18 shows the measured noise spectrum of the pixel output with and without the double modulation technique. The spectrum is obtained by the 8192 points fast Fourier transform (FFT) to the measured time-domain sequence of the pixel output. Only an unmodulated laser signal is applied. Using the double modulation technique as shown in Figure $18 \mathrm{~b}$ when compared with Figure 18a, in which the double modulation is not used, the low-frequency noise component, particularly $1 / \mathrm{f}$ noise, is suppressed effectively.

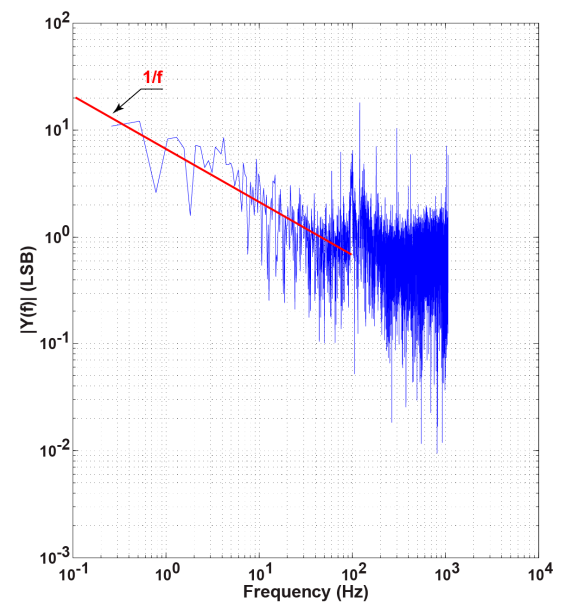

(a)

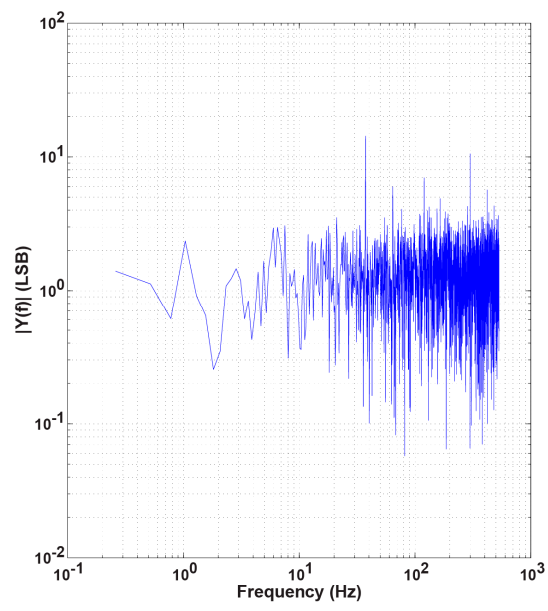

(b)

Figure 18. 1/f noise reduction effect of the double modulation: (a) without double modulation technique (b) with double modulation technique.

To show the effectiveness of the proposed circuit and the possibility of detecting a very small SRS effective signal included in a large offset component, the linearity of the pixel output to the AC signal power normalized to the DC power is measured as shown in Figure 19. In this measurement, the power of the DC laser is kept unchanged while the measurements are done by changing AC laser power only. A plot of the averaged values of six measurements is shown. The time taken for each measurement is about five minutes, which includes the manual changing of the filter. A good linearity for a power ratio from $10^{-2}$ to $10^{-5}$ is obtained. In the region of very small AC power $\left(<10^{-5}\right)$, the output signal is not stable. One of the reasons is that, in the range of $<10^{-5}$, the output signal from the pixels becomes very small, and, if it is smaller than 1 LSB, the signal after A/D conversion is much influenced by the nonlinearity of the A/D converter used.

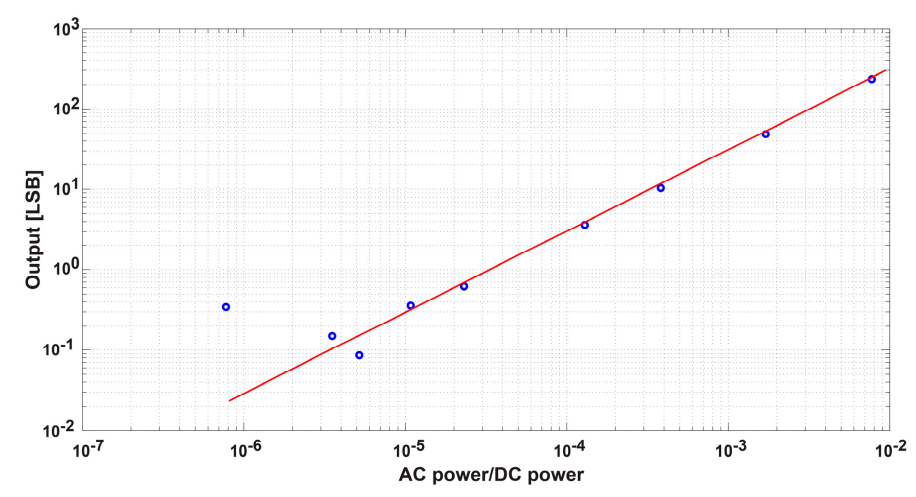

Figure 19. Small signal detection linearity. 
A sample of Benzonitrile prepared on a glass sample holder is used to confirm the effectiveness of the proposed lock-in pixel for SRS spectrum measurements. The measured SRS spectrum is shown in Figure 20. The measurement is currently performed manually with measurement time of about $15 \mathrm{~min}$. Automated measurement will be performed in future work where a trigger signal is sent from the sensor board at regular intervals to the laser system for wavelength tuning and capture. The figure also shows the spectrum simultaneously measured using coherent anti-stokes Raman spectroscopy (CARS) for comparison purposes. According to the standard Raman spectra [15], Benzonitrile should have a Raman shift peak at $2229.4 \mathrm{~cm}^{-1}$. Measured SRS spectrum using the SRS lock-in pixel shows a clear Raman shift peak at $2230 \mathrm{~cm}^{-1}$. In the spectrum measured by CARS, the Raman shift peak appears at $2226.5 \mathrm{~cm}^{-1}$. These differences are might be due to the distortion of CARS spectrum by the effect of the non-resonant background. The noises that appear in the SRS spectrum are partly due to the limitation of the A/D converter resolution as discussed earlier in this section. A higher resolution A/D converter will be used in future work to improve the linearity. Improvement of signal-to-noise ratio can be further enhanced by modification of both detector and circuit. The use of the array of sub-pixels to achieve a large area detector in this imager might have deteriorated the signal-to-noise ratio. A large area LEFM should improve the stability of the output and thus improve signal-to-noise ratio. Additionally, a higher analog gain of the lock-in amplifier will be implemented to further boost the signal-to-noise ratio. Although the Raman shift obtained by the SRS lock-in pixel is noisy, the possibility of Raman shift spectra measurement using the CMOS lock-in pixel is recognized by Figure 20.

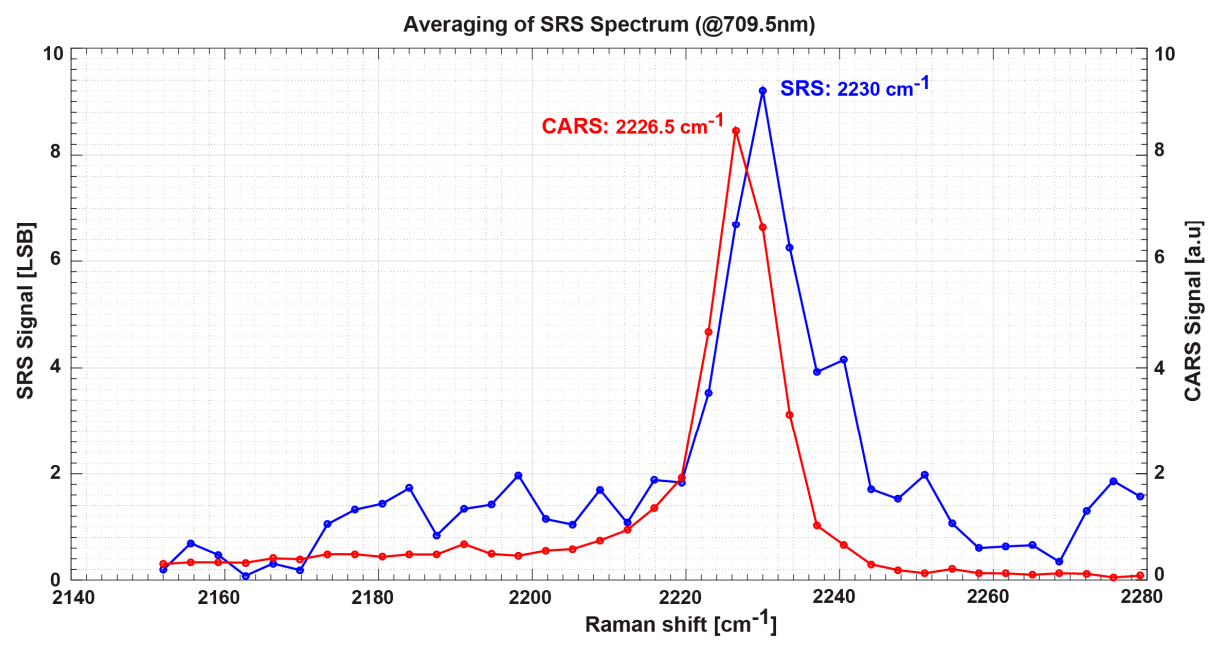

Figure 20. Benzonitrile Raman spectrum measured by coherent anti-stokes Raman spectroscopy (CARS) (red) and SRS (blue).

\section{Conclusions}

In this paper, a high-speed lock-in pixel technology for stimulated Raman scattering CMOS imagers is presented. The proposed lock-in pixel with a high-speed demodulator using a lateral electric field control of charge and the double modulation technique for reducing residual offset and low frequency noise components successfully detects small stimulated Raman scattering signals in huge offset due to the direct pump laser light. Careful setting of the phase delay between the LEFM demodulated output signal and the readout sampling clock suppresses the residual offset and prevents the saturation of the amplifier output by the offset when a large gain of the SC integrator is used. However, we acknowledge that it is challenging to implement the phase delay across an array of pixels. The implemented lock-in pixel successfully detects a small AC signal whose AC/DC power ratio is less than $10^{-5}$. The SRS spectrum of Benzonitrile has been successfully measured. These results are a significant advancement toward the goal of realizing the SRS CMOS image sensor. 
Acknowledgments: This research was supported by the Japan Science and Technology Agency (JST) under Collaborative Research Based on Industrial Demand "In Vivo Molecular Imaging: Towards Biophotonics Innovations in Medicine", and partly by the Japan Society for the Promotion of Science (JSPS) Grant-in-aid for Scientific Research (S), No. 25220905. This work was also supported by VLSI Design and Education Center (VDEC), the University of Tokyo in collaboration with Cadence Design Systems, Inc. and Mentor Graphics, Inc.

Author Contributions: De Xing Lioe, Kamel Mars, Shoji Kawahito, Keita Yasutomi and Keiichiro Kagawa conceived and designed the image sensor. Takahiro Yamada and Mamoru Hashimoto prepared the laser setup. All authors performed the experiment. De Xing Lioe and Kamel Mars carried out the data analysis and drafted the manuscript. Shoji Kawahito and Mamoru Hashimoto provided the overall guidance to the project. All authors have read and revised the manuscript.

Conflicts of Interest: The authors declare no conflict of interest.

\section{References}

1. Thomas, G.J. Raman Spectroscopy of Protein and Nucleic Acid Assemblies. Annu. Rev. Biophys. Biomol. Struct. 1999, 28, 1-27. [CrossRef] [PubMed]

2. Chan, J.W.; Taylor, D.S.; Zwerdling, T.; Lane, S.M.; Ihara, K.; Huser, T. Micro-Raman spectroscopy detects individual neoplastic and normal hematopoietic cells. Biophys. J. 2006, 90, 648-656. [CrossRef] [PubMed]

3. Van Manen, H.J.; Kraan, Y.M.; Roos, D.; Otto, C. Single-cell Raman and fluorescence microscopy reveal the association of lipid bodies with phagosomes in leukocytes. Proc. Natl. Acad. Sci. USA 2005, 102, 10159-10164. [CrossRef] [PubMed]

4. Evans, C.L.; Xie, X.S. Coherent Anti-Stokes Raman Scattering Microscopy: Chemical Imaging for Biology and Medicine. Annu. Rev. Anal. Chem. 2008, 1, 883-909. [CrossRef] [PubMed]

5. Yu, Y.; Ramachandran, P.V.; Wang, M.C. Shedding new light on lipid functions with CARS and SRS microscopy. Biochim. Biophys/ Acta (BBA) Mol. Cell Biol. Lipids 2014, 1841, 1120-1129. [CrossRef] [PubMed]

6. Day, J.P.R.; Domke, K.F.; Rago, G.; Kano, H.; Hamaguchi, H.O.; Vartiainen, E.M.; Bonn, M. Quantitative Coherent Anti-Stokes Raman Scattering (CARS) Microscopy. J. Phys. Chem. B 2011, 115, 7713-7725. [CrossRef] [PubMed]

7. Freudiger, C.W.; Min, W.; Saar, B.G.; Lu, S.; Holtom, G.R.; He, C.; Tsai, J.C.; Kang, J.X.; Xie, X.S. Label-Free Biomedical Imaging with High Sensitivity by Stimulated Raman Scattering Microscopy. Science 2008, 322, 1857-1861. [CrossRef] [PubMed]

8. Nandakumar, P.; Kovalev, A.; Volkmer, A. Vibrational imaging based on stimulated Raman scattering microscopy. New J. Phys. 2009, 11, 033026. [CrossRef]

9. Berto, P.; Andresen, E.R.; Rigneault, H. Background-Free Stimulated Raman Spectroscopy and Microscopy. Phys. Rev. Lett. 2014, 112, 053905. [CrossRef] [PubMed]

10. Rock, W.; Bonn, M.; Parekh, S.H. Near shot-noise limited hyperspectral stimulated Raman scattering spectroscopy using low energy lasers and a fast CMOS array. Opt. Express 2013, 21, 15113-15120. [CrossRef] [PubMed]

11. Spirig, T.; Seitz, P. The Lock-in CCD—Two-dimensional synchronous detection of light. IEEE J. Quantum Elec. 1995, 31, 1705-1705. [CrossRef]

12. Kawahito, S.; Baek, G.; Li, Z.; Han, S.-M.; Seo, M.-W.; Yasutomi, K.; Kagawa, K. CMOS lock-in pixel image sensors with lateral electric field control for time-resolved imaging. In Proceedings of the International Image Sensor Workshop, Snowbird, UT, USA, June 2013; pp. 316-364.

13. Cahydi, H.; Iwatsuka, J.; Minamikawa, T.; Niioka, H.; Araki, T.; Hashimoto, M. Fast spectral coherent anti-Stokes Raman scattering microscopy with high-speed tunable picosecond laser. J. BioMed. Opt. 2013, 18, 096009. [CrossRef] [PubMed]

14. Minamikawa, T.; Hashimoto, M.; Fujita, K.; Kawata, S.; Araki, T. Multi-focus excitation coherent anti-Stokes Raman scattering (CARS) microscopy and its applications for real-time imaging. Opt. Express 2009, 17, 9526-9536. [CrossRef] [PubMed]

15. Standard Spectra. Available online: http://www.chem.ualberta.ca/ mccreery/ramanmaterials.html (accessed on 15 October 2014).

(C) 2016 by the authors; licensee MDPI, Basel, Switzerland. This article is an open access article distributed under the terms and conditions of the Creative Commons Attribution (CC-BY) license (http://creativecommons.org/licenses/by/4.0/). 